\title{
Scintigraphic assessment of vascularity and blood-tissue barrier of human brain tumours
}

\author{
D. F R O N T
}

From the Division of Nuclear Medicine and Departments of Oncology, Neurology, and Neurosurgery, Rambam Medical Center and The School of Medicine of the Technion, Haifa, Israel

SUMMARY Assessment of vascularity and blood-tissue barrier was performed by sequential scintigraphy in 43 patients with brain tumours. The blood-tumour barrier was evaluated by use

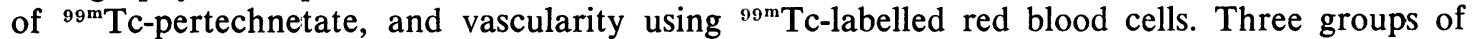
tumours were found: tumours with low vascularity and permeable barrier, tumours with high vascularity and permeable barrier, and tumours with low vascularity and relatively impermeable barrier. The first group indicates that when vessels are permeable, there may be a rapid penetration of large amounts of pertechnetate into the tumour even when vascularity is not increased. In the other two groups penetration of pertechnetate into the tumour is affected by vascularity, as it determines the total area where passage of the radiopharmaceutical takes place. It is suggested that the permeability of the blood-tumour barrier and the amount of vascularity may have an effect on the success of chemotherapy in brain tumours.

Penetration of pharmaceuticals into the tissue of brain tumours has been a subject of interest to a number of investigators, and a variety of directions were emphasised (Matthews and Molinaro, 1963; Tator et al., 1965; Bakay, 1967, 1969, 1970; Baum, 1971; Brightman et al., 1971; Penning et al., 1971, 1973; Gado et al., 1975a, b; Penning and Front, 1975).

Two main factors emerge as governing the passage from blood into the tissue of tumours-the vascularity of the tumour and the permeability of the blood-tissue barrier. In previous reports the role of the blood-tumour barrier in the uptake of pertechnetate was assessed (Penning et al., 1973; Penning and Front, 1975). The role of tumour vascularity, however, could be deduced only indirectly. Radionuclide angiography and 'blood pool' studies with pertechnetate were the scintigraphic methods used for assessment of tumour vascularity. They are neither sensitive nor accurate. In radionuclide angiography, the short time interval between the frames allows only for poor statistics of the scintigrams, and only tumours with marked vascularity are clearly evident. In the blood pool studies pertechnetate, in many cases, is already penetrating into the tissue of the tumour.

Address for correspondence and reprint requests : Dr D. Front, Division of Nuclear Medicine, Rambam Medical Center, Haifa, Israel.

Accepted 21 July 1977
The recent development of a method of labelling red blood cells with ${ }^{99 \mathrm{~m}} \mathrm{TC}$, achieving a very high tagging efficiency, creates an intravascular agent suitable for assessment of vascularity.

In this paper I compare sequential scintigraphy of brain tumours performed with ${ }^{99^{\mathrm{m}} \mathrm{Tc}-}$ pertechnetate for evaluation of the permeability of tumour vessels, with sequential scintigraphy performed with labelled red blood cells, and for tumour vascularity.

\section{Methods}

Forty-three patients were studied first using ${ }^{99^{\mathrm{m}}} \mathrm{Tc}$ pertechnetate and 72 hours later using ${ }^{99^{\mathrm{m}} \mathrm{Tc}-}$ labelled red blood cells. An Anger type gamma camera and a parallel hole low energy collimator were used. Scintigrams were recorded on $\mathrm{x}$-ray film using an electronic programmer and imager. The same protocol was followed in both pertechnetate and labelled red blood cells studies. Radionuclide angiography was performed after the intravenous injection of $15 \mathrm{mCi}$ of the pharmaceutical, and 16 pictures were recorded with exposure time of three seconds. A four-view study, described as the 'blood pool study', collecting 400000 counts per scintigram, immediately followed. The final static study, using the same number of counts and views, was done after two 
hours. Labelling of red blood cells was performed using a Soreq kit (Soreq Nuclear Plants, Yavne, Israel) which ensures a tagging efficiency of $97 \%$. Strict quality control was kept in the studies with regard to the existence of free pertechnetate. Labelling was checked before studies, by chromatography, and in the final static scintigrams, by looking for uptake in the parotid and other salivary glands. Any such uptake was considered to indicate free pertechnetate, and the study was discarded.

Patients also underwent a neuroradiological survey consisting of all or a combination of radiocontrast angiography, pneumoencephalography, and computed tomography. In all primary brain tumours there was histological proof as to the nature of the tumour. In the metastatic tumours there was histological proof of the primary cancer. Investigations and clinical course were compatible with brain metastasis.

\section{Results}

EARLY AND MARKEI) VISUALISATION USING PERTECHNETATE ANI) SLIGHT OR NO VISUALISATION USING LABELLED RED BLOOD CELLS (FIG. 1)

Using pertechnetate, the tumours in this group were visible already in the radionuclide angi-

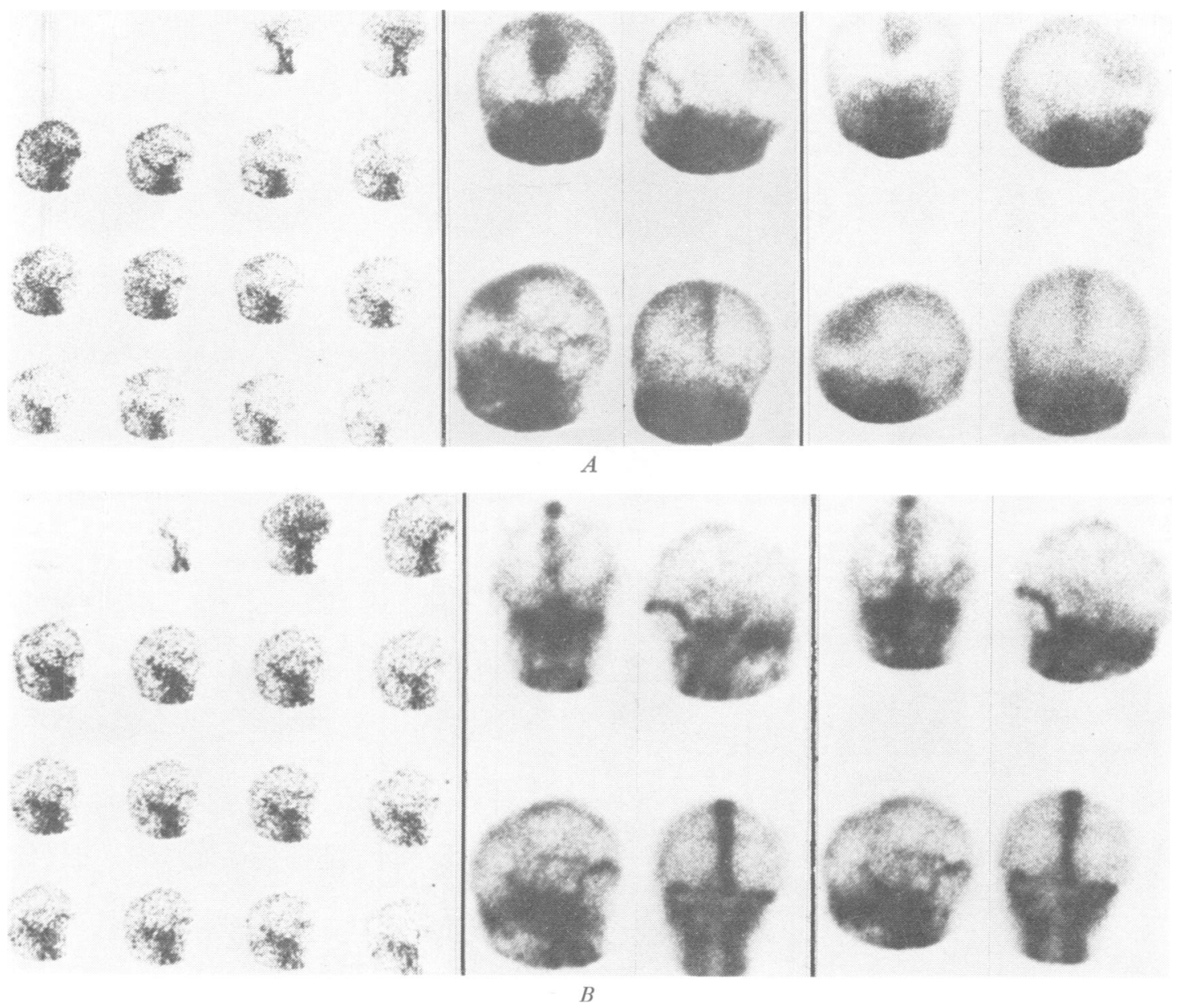

Fig. 1 Low vascularity and high permeability of the blood-tumour barrier in a case of meningioma. "'T(-pertechnetate study. A shows radionuclide angiography (left), blood pool study (centre), and static study (right). Marked and early visualisation of abnormal radioactivity in frontal parasagittal region. $B$ shows "9" Tc-labelled red blood cells study in the same order. Lesion is not demonstrated in radionuclide angiography, and only faintly visible in static studies. 
ography. There was marked and persistent demonstration of the tumour in the blood pool and static studies. Radionuclide angiography with labelled red blood cells did not show the tumour, and it wois only faintly visible or not visible at all in the blood pool and static studies. This pattern is interpreted as indicating low vascularity of the tumour, but high permeability of the blood vessels.

EARLY VISULISATION USING BOTH PERTECHNETATE AND LABELLED RED BLOOD CELLS (FIG. 2)

In this pattern the tumour was visible with both pertechnetate and labelled red blood cells already in the radionuclide angiography. Visualisation persisted for the rest of the study. This pattern indicates high vascularity and high permeability of the blood-tumour barrier.

LATE VISUALISATION USING PERTECHNETATE AND NO VISUALISATION WITH LABELLED RED BLOOD CELLS (FIG. 3)

In this group the tumour was visible only faintly and was not visible at all with labelled red blood cells; nor was it demonstrated by radionuclide angiography and blood pool study with pertechnetate. Visualisation was achieved only in the late static study two hours after the intravenous injections. This pattern indicates low vascularity and relatively low permeability of the blood vessels.

The patterns of visualisation in various brain tumours are summarised in the Table.

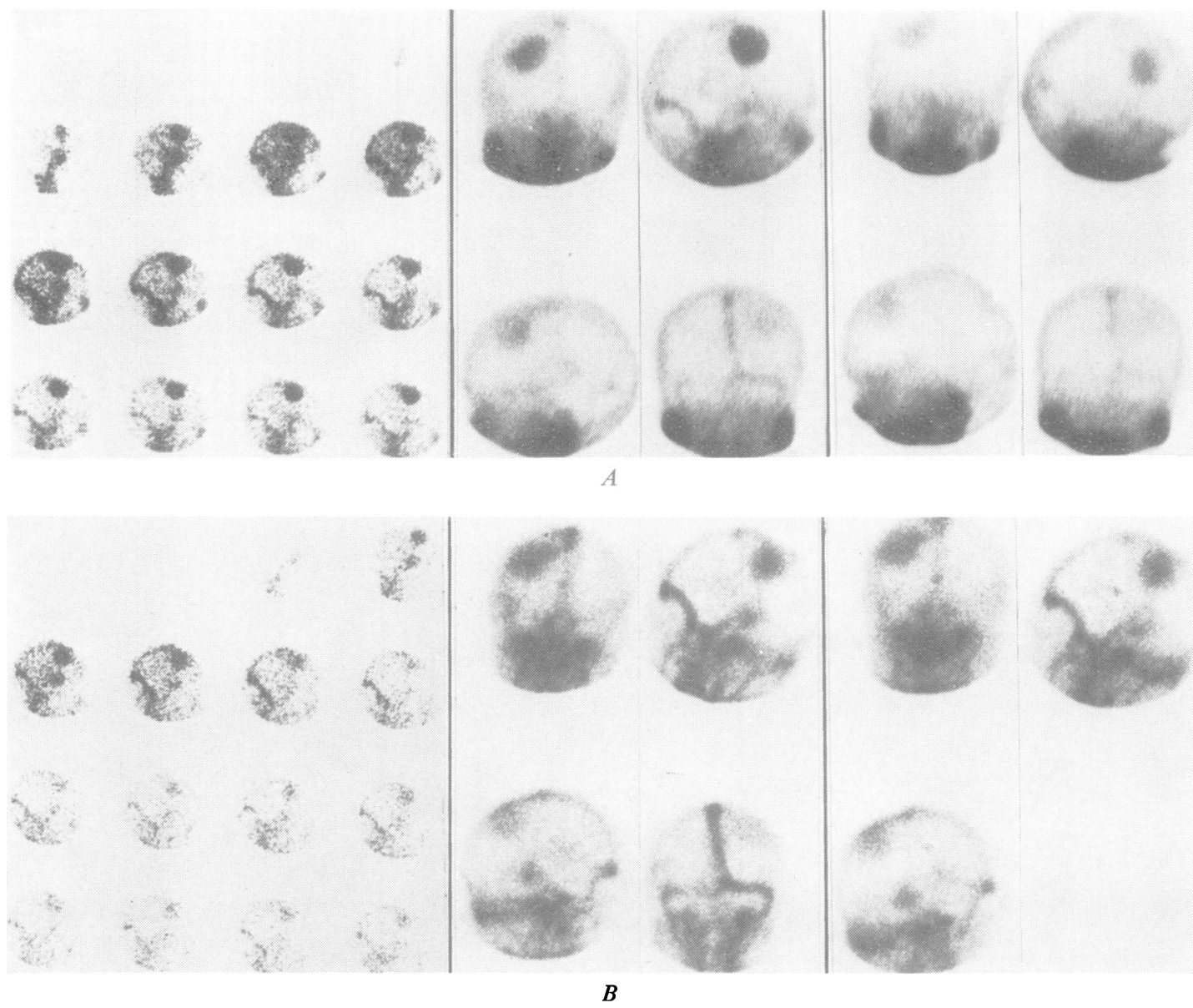

Fig. 2 High vascularity and high permeability of the blood-tumour barrier in a case of meningioma${ }^{9 y}$ Tc-pertechnetate study. A shows radionuclide angiography (left), blood pool study (centre), and static study (right). Marked and early visualisation of lesion. B shows ${ }^{99 m}$ Tc-labelled red blood cells study in the same order. Marked visualisation of the lesion in all studies. 


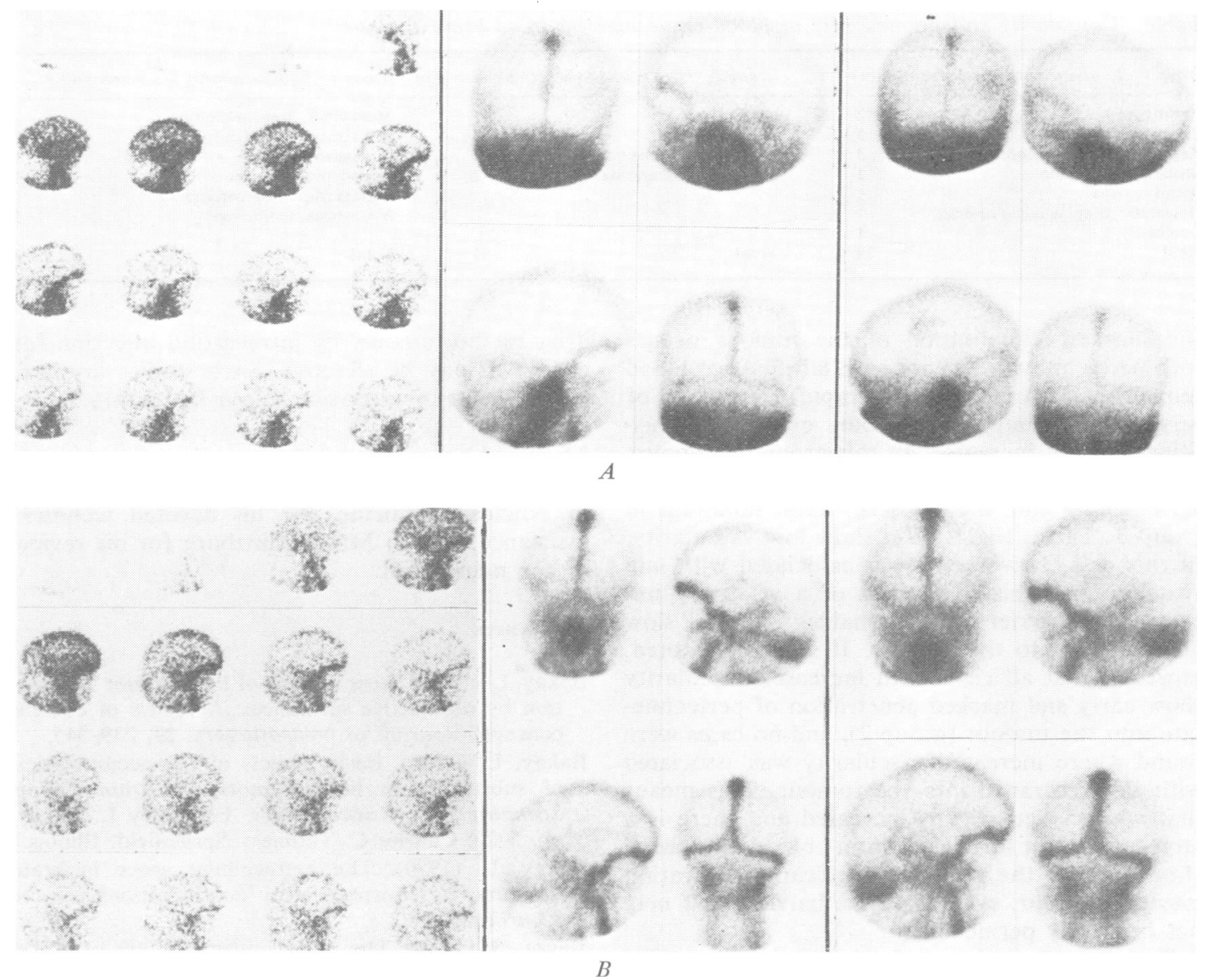

Fig. 3 Low vascularity and permeability of the blood-tumour barrier in a case of ependymoblastoma${ }^{99 m}$ Tc-pertechnetate study. A shows radionuclide angiography (left), blood pool study (centre), and static study (right). Abnormal accumulation of radioactivity deep in the left parietal region in the static study. No abnormality in the radionuclide angiography and blood pool study. B shows ${ }^{99 m}$ Tc-labelled red blood cells study in the same order. No abnormality.

\section{Discussion}

Sequential brain scintigraphy shows that pertechnetate enters various brain tumours at different rates (Gates et al., 1971; Handa, 1971; Penning et al., 1971; Ramsey and Quinn, 1972; Sheldon et al., 1975). Some tumours are visualised early, some late, and others not at all. It is necessary to postulate some kind of barrier between the blood and the tissue of the tumour-the blood-tumour barrier (Penning et al., 1973; Gado et al., 1975a). The question arises as to the nature of this barrier. It may reflect the amount of tumour vascularity which determines the area for transport of pharmaceuticals from blood into the tissue (Long, 1973). Increase in this area results in higher penetration of pharmaceuticals into the tumour tissue and vice versa. In such cases penetration of radiopharmaceuticals would be totally dependent on vascularity. Alternatively the barrier may indicate different degrees of permeability in vessels of different tumours which is to a greater or lesser extent independent of the vascularity. A logical solution to this problem in vivo, is to compare tumour permeability to pertechnetate, as shown by sequential scintigraphy, against tumour vascularity demonstrated by scintigraphy using labelled red blood cells.

The results of this study show that the degree of penetration of the radiopharmaceutical may be independent of the amount of vascularity of the tumour at hand. In group 1 (Table, Fig. 1), early 
Table Vascularity and permeability of blood-tissue barriers in 43 brain tumours

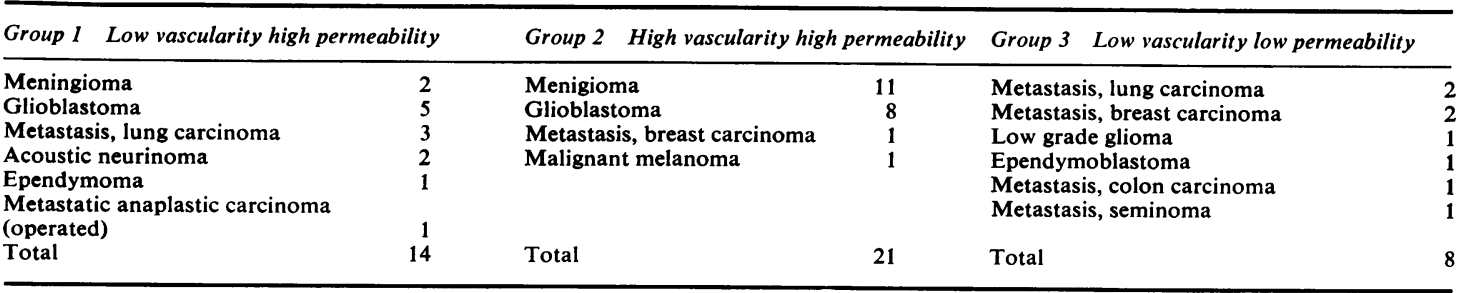

and marked visualisation of the tumour occurs with pertechnetate but not with labelled red blood cells. This is an indication of rapid penetration of pertechnetate into the tumour, even when vascularity is not increased. In this group of tumours the blood-tumour barrier is determined by the permeability of the vessels. The tumours in group 3 (Table, Fig. 3) also show low vascularity. In this case, however, it was associated with late visualisation. This is a result of a relatively impermeable barrier which enables only a slow penetration into the tumour. It should be noted, however, that all cases with increased vascularity show early and marked penetration of pertechnetate into the tumour (group 2), and no cases were found where increased vascularity was associated with slow entrance into the tumour. This means that when vascularity is increased and there is a large area for passage from blood to tissue (Long, 1973), the result is significant penetration into the tumour, even when the barrier itself may not be highly permeable.

Brain tumours have blood vessels that differ anatomically from normal vessels of the brain in that they have open endothelial cell junctions, gaps between endothelial cells, and fenestrations in capillary endothelial membranes (Long, 1970, 1973; Tani et al., 1974). These are the anatomical paths by which pharmaceuticals enter the extracellular space of tumours (Long, 1973; Gado et al., 1975a, b; Penning and Front, 1975).

If chemotherapeutic drugs penetrate tumours through the same anatomical channels as pertechnetate, it is reasonable to suggest, but still remains to be proved, that the barrier to drugs acts along the same lines as that of pertechnetate. The detailed assessment of the blood-tumour barrier to a labelled drug, and its relation to vascularity, may have a value in planning effective chemotherapy. It is probably of no use to treat tumours with low vascularity and low permeability as only a small amount of the drug will reach and penetrate the tumour. On the other hand, in tumours with abundant vascularity and high permeability the delivery of large amounts of the drug to the tumour, by intracarotid injection for example, may be effective. Such studies are currently under investigation at our laboratory.

The author expresses his appreciation to $\mathrm{Mr}$ Nabil Maschuur, Chief Technician of the Division of Nuclear Medicine, for his devoted technical assistance, and to Mr G. Ginzburg for his review of the manuscript.

\section{References}

Bakay, L. (1967). Basic aspects of brain tumor localization by radioactive substances. A review of current concepts. Journal of Neurosurgery, 27, 239-345.

Bakay, L. (1969). Basic aspects of the accumulation of substances in brain tumors. In Brain Tumor Scanning with Radioisotopes. Edited by L. Bakay, pp. 5-20. Charles C. Thomas: Springfield, Illinois.

Bakay, L. (1970). The extracellular space in brain tumours I: Morphological considerations. Brain, 93, 693-698.

Baum, S. (1971). The site of accumulation of ${ }^{19 m}$ Tcsodium pertechnetate in brain tumors. Radiology. 99, 153-155.

Brightman, M. W., Reese, T. S., and Vick, N. A. (1971). A mechanism underlying the lack of a blood-brain barrier to peroxidase in virally induced brain tumors. Journal of Neuropathology and Experimental Neurology, 30, 139-140.

Gado, M. H., Phelps, M. E., and Coleman, R. E. (1975a). An extravascular component of contrast enhancement in cranial computed tomography. Part I: the tissue-blood ratio of contrast enhancement. Radiology, 117, 589-593.

Gado, M. H., Phelps, M. E., and Coleman, R. E. (1975b). An extravascular component of contrast enhancement in cranial computed tomography. Part II: contrast enhancement and the blood-tissue barrier. Radiology, 117, 595-597.

Gates, G. F., Dore, E. K., and Toplin, G. V. (1971). Interval brain scanning with sodium pertechnetate${ }^{99 \mathrm{~m}} \mathrm{Tc}$ for tumor detectability. Journal of the American Medical Association, 215, 85-88.

Handa, J. (1971). Sequential brain imaging as an aid in understanding disease etiology. Seminars in Nuclear Medicine, 1, 56-69. 
Long, D. M. (1970). Capillary ultrastructure and the blood brain barrier in human malignant brain tumors. Journal of Neurosurgery, 32, 127-144.

Long, D. M. (1973). Vascular ultrastructure in human meningiomas and schwannomas. Journal of Neurosurgery, 38, 409-419.

Matthews, C. M. E., and Molinaro, G. (1963). A study of the relative value of radioactive substances used for brain tumour localisation and for the mechanism of brain tumour concentration. Uptake in transplantable fibrosarcoma, brain and other organs in the rat. British Journal of Experimental Pathology, 44, 260-277.

Penning, L., Front, D., and Beekhuis, H. (1971). Differentiation of brain lesions by sequential gamma camera studies. Journal of the Neurological Sciences, 14, 1-13.

Penning, L., Front. D., Bechar, M., Go, K. G., and Rodermond, J. M. (1973). Factors governing the uptake of pertechnetate by human brain tumours:
A scintigraphic study. Brain, 96, 225-234.

Penning, L., and Front, D. (1975). Brain Scintigraphy. A Neuroradiological Approach, pp. 43-49. Excerpta Medica: Amsterdam.

Ramsey, R. G., and Quinn, J. L. (1972). Comparison of accuracy between initial and delayed ${ }^{: y_{n}} \mathrm{Tc}$ pertechnetate brain scans. Journal of Nuclear Medicine, 13, 131-134.

Sheldon, J. J., Smoak, W. M., and Serafini, A. N. (1975). Dynamic scintigraphy of primary and secondary malignant intracranial neoplasms. Radiology, 114, 373-380.

Tani, E., Ikeda, K., and Kudo, S. (1974). Fenestrated vessels in human hemangioblastoma. Journal of Neurosurgery, 40, 690-705.

Tator, C. H., Morcley, T. P., and Olszewsky, J. (1965). A study of the factors responsible for the accumulation of RIHSA by intracranial tumors and other lesions. Journal of Neurosurgery, 22, 60-76. 